\title{
Isospin Non-Conservation in Shell Model Approach and Applications*
}

\author{
Yi Hua Lam $^{1, * *}$ and Nadezda A. Smirnova ${ }^{2, * * *}$ \\ ${ }^{1}$ Key Laboratory of High Precision Nuclear Spectroscopy, Institute of Modern Physics, Chinese \\ Academy of Sciences, Lanzhou 730000, China \\ ${ }^{2}$ CENBG, CNRS/IN2P3 and Université de Bordeaux, Chemin du Solarium, 33175 Gradignan cedex, \\ France
}

\begin{abstract}
Up to now, empirical shell-model Hamiltonians for valence space calculations provide the most accurate description of the low-energy spectra and individual transitions of $s d$ - and $p f$-shell nuclei. These features made them of particular importance for the description of the isospin-symmetry-breaking phenomena, such as energy splitting of the isobaric multiplets or isospin-forbidden transition rates. In this contribution, we demonstrate the applications of a recently constructed isospin non-conserving (INC) Hamiltonian in $s d$ shell [Lam et al. Phys. Rev. C 87, 054304 (2013)]. First, we explore the partial decay scheme of ${ }^{24} \mathrm{Si}$ and discuss the states affected by the Thomas-Ehrman shift. Second, we show the accuracy of the INC Hamiltonian for the description of the mirror energy differences.
\end{abstract}

\section{Introduction}

The concept of isospin symmetry significantly simplifies nuclear many-body calculations and enables us to understand the structure of nuclei in the vicinity of the $N=Z$ line. The isospin symmetry implies that a nuclear many-body Hamiltonian is invariant under all rotations in isospin space. However, it is only an approximate symmetry of atomic nuclei due to the Coulomb interaction between protons and a slight neutron-proton mass difference. Modern experiments at radioactive ion beam facilities are capable to bring accurate information on the isospin-symmetry breaking in various phenomena, ranging from the splittings of the isobaric multiplets to rates of isospin-forbidden decays. It is therefore a challenge for a microscopic nuclear theory to describe the experimental observations, making thus a link to the isospin properties of the nucleon-nucleon interaction.

In this contribution, we use an $s d$-shell INC Hamiltonian [1], which is based on the isospin-conserving USDB Hamiltonian [2] and includes (i) a two-body Coulomb potential, normalized for the short-range correlations by the unitary correlation operator method [3], (ii) an effective charge-dependent two-body term of nuclear origin, and (iii) isovector singleparticle energies. The strengths of those charge-dependent terms have been determined by

\footnotetext{
*The work is supported by the National Natural Science Foundation of China under Grant No. 11775277, the financial support to the "Théorie-ISB" project (IN2P3/CNRS, France), and the Ministry of Foreign Affairs and International Development of France in the framework of PHC Xu GuangQi 2015 under project No. 34457VA.

**e-mail: lamyihua@gmail.com

***e-mail: smirnova@cenbg.in2p3.fr
} 
a least-squares fit to reproduce the experimental isovector and isotensor coefficients of the isobaric-multiplet-mass equation (IMME) [4]. The INC Hamiltonian is named cdUSDB.

Two applications are discussed below. One is related to a beta $(\beta)$-delayed proton decay of ${ }^{24} \mathrm{Si}$, while the second part is devoted to the description of the mirror energy differences in $s d$-shell $T=1 / 2$ nuclei. All calculations have been performed with the NuShellX@MSU shell-model code [5]. The results are compared with the existing experimental data.

\section{Partial-decay scheme of 24Si.}

The $\beta$-delayed particle-radioactivity is a powerful tool in studies of proton-rich nuclei [6], since it provides us with direct information on decay properties of excited states in exotic nuclei. In the context of the isospin-symmetry violation, we are particularly interested in the decay of the isobaric analogue state (IAS) in a daughter nucleus, which is often characterized by competing electromagnetic and isospin-forbidden particle decay channels, important for theoretical understanding [7, 8].

The decay of ${ }^{24} \mathrm{Si}$ has been a subject of intensive experimental and theoretical studies in recent decades [9-12]. The $\beta_{+}$decay of ${ }^{24} \mathrm{Si}$ populates, besides the IAS, $0^{+}, T=2$, a number of $1^{+}, T=1$ states in ${ }^{24} \mathrm{Al}$. All these states manifest themselves by gamma decay and/or by proton emission, populating the low-lying $T=1 / 2$ states in ${ }^{23} \mathrm{Mg}$. Obviously, the proton emission from the IAS is forbidden by the isospin symmetry. Thus, the measurements of the proton branching ratio give information on the existence of the isospin-mixing in the IAS. Besides, the analysis of $\beta-\gamma$ coincidences in the decay of ${ }^{24} \mathrm{Si}[11,12]$ has provided information on the Gamow-Teller (GT) branches to the two lowest $1^{+}$states. The interest is due to the fact that the second excited $1^{+}$state is known to be strongly shifted compared to its mirror state in ${ }^{24} \mathrm{Na}$, suggesting the important role of the weakly bound $1 s_{1 / 2}$ orbital (Thomas-Ehrman effect).

The purpose of the present work is to reproduce the main features of the decay scheme within the shell model. Our preliminary results are summarized in two tables inserted in Fig.1 near the experimental partial decay scheme. In addition to cdUSDB, we use the INC term established by Ormand and Brown [13], on top of the USDB Hamiltonian (OBUSDB). The GT operator is quenched by a standard factor $q=0.776$ [14]. The half-life of ${ }^{24} \mathrm{Si}$ is about $20 \%$ larger than the experimental value and the distribution of the GT strength is in robust agreement with the experimental data. It is seen from the tables on Fig.1, that both interactions predict very similar GT branching ratios as it is the isospin-invariant USDB Hamiltonian which is mainly responsible for that. The theoretical results reproduce very well the GT feeding of the two lowest $1^{+}$states, however, the numbers for the next two $1^{+}$states seem to be inverted with respect to the experiment.

We also employed these two INC Hamiltonians to describe the ratio of the $\mathrm{B}(\mathrm{GT})^{+}$to $\mathrm{B}(\mathrm{GT})^{-}$strengths to the two lowest $1^{+}$states in mirror nuclei ${ }^{24} \mathrm{Al}$ and ${ }^{24} \mathrm{Na}$, as well as the ratio of $\mathrm{B}(\mathrm{GT})^{+}$to the $1_{1}^{+}$and $1_{2}^{+}$states in ${ }^{24} \mathrm{Al}$, as shown in Table 1 . It is remarkable that the theoretical $\mathrm{B}(\mathrm{GT})$-values and their ratios are very close to the experimental values. As we mentioned in the introduction, the second $1^{+}$state in ${ }^{24} \mathrm{Al}$ manifests a noticeable ThomasEhrman effect: its excitation energy is $258 \mathrm{keV}$ lower than of its mirror in ${ }^{24} \mathrm{Na}$. Indeed, the $1 s_{1 / 2}$ orbital occupation is about twice larger than that in the $1_{1}^{+}$state. The detailed analysis of the mirror (a)symmetry will be published elsewhere [15].

In addition, we have analyzed the $\beta$-delayed proton emission from ${ }^{24} \mathrm{Si}$. The branching ratios of the isospin-forbidden proton decay of the IAS are presented in the table inserted in Fig. 1 (left part). It is evident that two Hamiltonians produce different branching ratios, due to the different parameterizations of the INC term used. The branching ratios from OBUSDB are in a slightly better agreement with the recent values published by Ichikawa et al [11]. 
Both interactions predict about $11-12 \%$ branch to the lowest $5 / 2^{+}$state in ${ }^{23} \mathrm{Mg}$, which has not yet been seen experimentally. It would be interesting to have more refined experimental data to be able to compare with the theory in more detail.

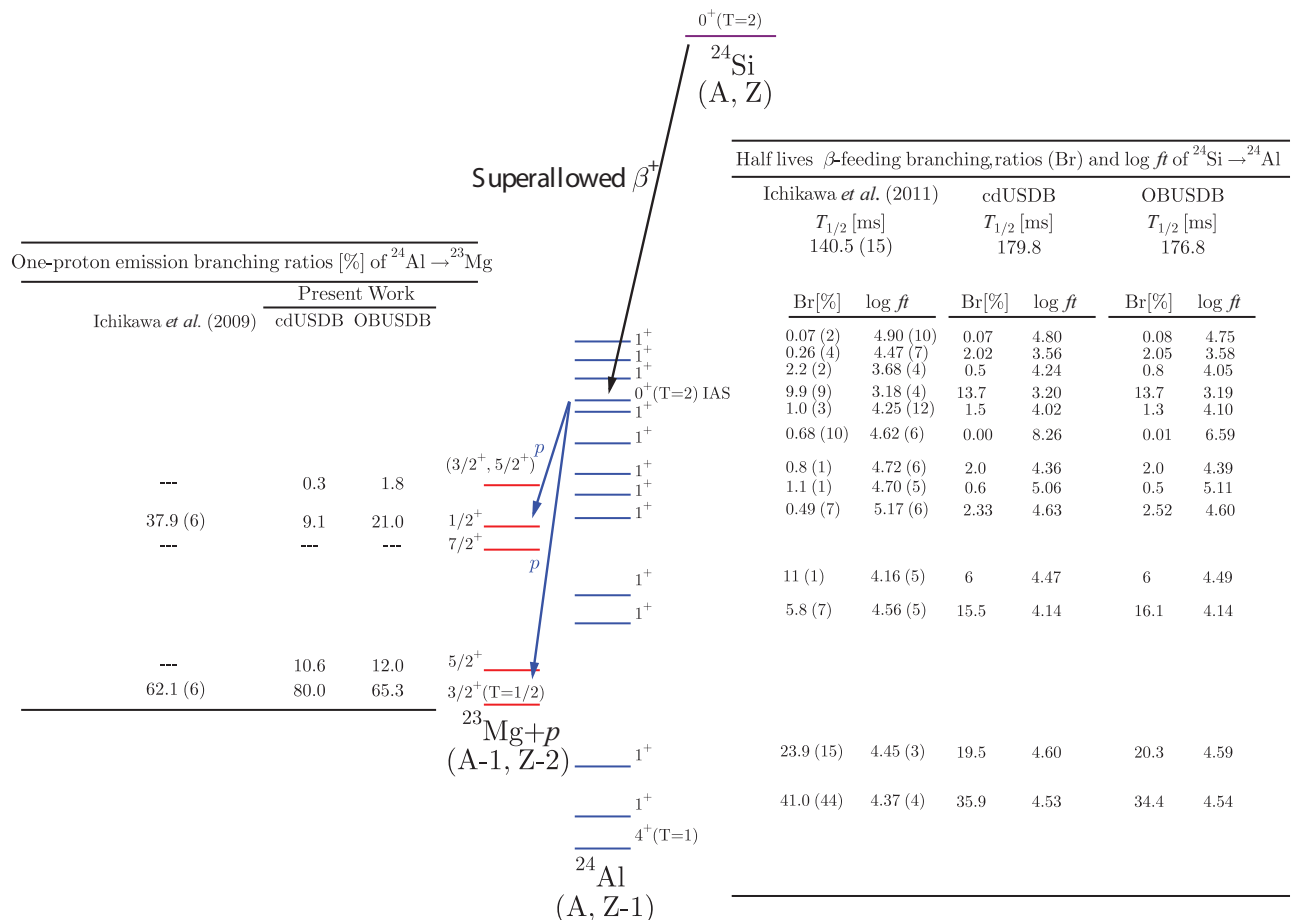

Figure 1. Partial-decay scheme of ${ }^{24} \mathrm{Si}$.

Table 1. Gamow-Teller strengths of ${ }^{24} \mathrm{Si} \rightarrow{ }^{24} \mathrm{Al}$ and ${ }^{24} \mathrm{Ne} \rightarrow{ }^{24} \mathrm{Na}$ for the $1_{1}^{+}$and $1_{2}^{+}$states.

\begin{tabular}{lllllllll}
\hline \hline & \multicolumn{3}{c}{${ }^{24} \mathrm{Si} \rightarrow{ }^{24} \mathrm{Al}$} & & \multicolumn{3}{c}{${ }^{24} \mathrm{Ne} \rightarrow{ }^{24} \mathrm{Na}$} & \multicolumn{3}{c}{$B\left(\mathrm{GT}^{+}\right) / B\left(\mathrm{GT}^{-}\right)$} \\
& $B\left(\mathrm{GT}^{+}\right)^{1^{+}}$ & $B\left(\mathrm{GT}^{+}\right)^{1_{2}^{+}}$ & ratio & $B\left(\mathrm{GT}^{-}\right)^{1_{1}^{+}}$ & $B\left(\mathrm{GT}^{-}\right)^{1_{2}^{+}}$ & ratio & $1_{1}^{+}$ & $1_{2}^{+}$ \\
\hline Ref. [12] & $0.17(2)$ & $0.14(1)$ & $1.21(17)$ & $0.167(4)$ & $0.155(9)$ & $1.077(68)$ & $1.02(12)$ & $0.90(8)$ \\
OBUSDB & 0.1631 & 0.1463 & 1.12 & 0.1832 & 0.1586 & 1.155 & 0.89 & 0.92 \\
cdUSDB & 0.1936 & 0.1665 & 1.16 & 0.2116 & 0.1815 & 1.166 & 0.91 & 0.92 \\
\hline
\end{tabular}

\section{Mirror energy difference}

The other study is focused on excitation-energy differences between excited states of mirror nuclei, known as mirror energy difference (MED). In the last decades, a good amount of accurate experimental information has become available on the MEDs in $s d$ - and $p f$-shell nuclei and it has been recognized that the MEDs are a very sensitive probe to the details of the INC Hamiltonian (for review see Refs. $[16,17]$ and references therein). Each MED is caused by the Coulomb interactions between protons and by a smaller contribution from effective charge-dependent interaction of nuclear origin. Analyzed as a function of the excitation energy and of the angular momentum, the MEDs contain valuable information on the 
dynamical changes in the structure of the excited states and allow us to understand the role of the broken isospin symmetry in quantum many-body systems.

The MED is defined as a difference in excitation energies of the mirror states and may be considered as a function of the angular momentum $J$ and isospin $T[16,17]$,

$$
\operatorname{MED}_{J, T}=E_{J, T, T_{z}=-T}^{*}-E_{J, T, T_{z}=T}^{*}
$$

where $T=\left|T_{z}\right|$ as we only study a set of excited states of the lowest isospin. For $T=1 / 2$ multiplets, the MED can be related to the differences of the isovector $(b)$ coefficient of IMME, $\operatorname{MED}_{J, 1 / 2}=-\Delta b_{J}$, where $\Delta b_{J}$ is the difference of the $b$ coefficients between the excited state of spin $J$ and the ground state. The magnitude of these energy differences is rather small compared to the total contribution of the Coulomb energy to the nuclear mass of a given nucleus, and to the splitting of IASs. Nevertheless, this energy difference carries important information on a subtle charge asymmetry of the nuclear interaction. The measured MED vary typically from a few tens of $\mathrm{keV}$ and up to about $120 \mathrm{keV}$ for $s d$-shell $T=1 / 2$ doublets. Figures 2 and 3 show the available experimental data on MEDs of $T=1 / 2$ yrast states in $A=21[18]$ and $A=23[19,20]$.

It was found by Zuker et al. [21], see also [16], that an addition of a specific $J$-dependent matrix element is necessary to reproduce the MED trends in $p f$-shell nuclei. In the present study, we show that with the INC Hamiltonians described above we reasonably reconstruct the MEDs of yrast states of $s d$-shell $T=1 / 2$ multiplets. The results from the cdUSDB and OBUSDB interactions for $A=21$ and $A=23$ are shown in Figs. 2 and 3. We notice that in $A=21$, the OBUSDB results are in very good agreement with the experiment, except for $7 / 2^{+}$state. The results obtained with cdUSDB exhibit some more discrepancies for high-spin states. Contrary, in the $A=23$ case, both interactions well reproduce the experimental trend with cdUSDB being the best. We remark that the MED trends of a few others $s d$-shell mirror pairs are robustly reproduced as well, e.g., in $T=1 / 2, A=23,29,31$, and 35. Nevertheless, further work is needed to tune the INC terms of the Hamiltonian to improve the predictive power for MEDs of $T=1 / 2, A=21,25,27$, and 33. A detailed study of the whole series of multiplets and a further analysis of the agreement will be published elsewhere. We hope that this work will shed more light on the structure of the INC Hamiltonian, in particular, on the most optimal form of the charge-dependent terms of nuclear origin.

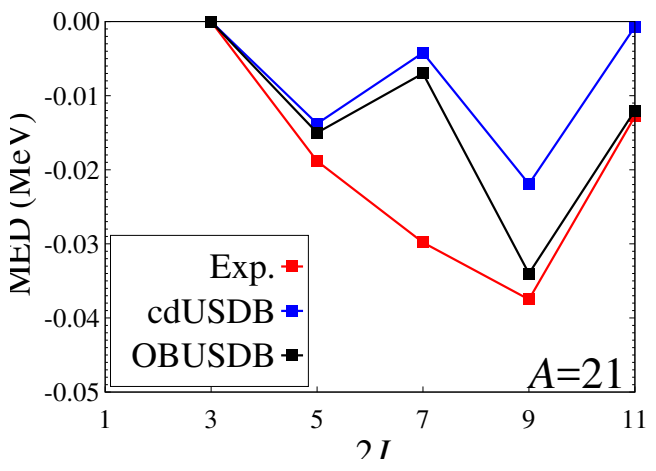

Figure 2. The MED of mass $A=21$. Experimental data are deduced from NNDC [18].

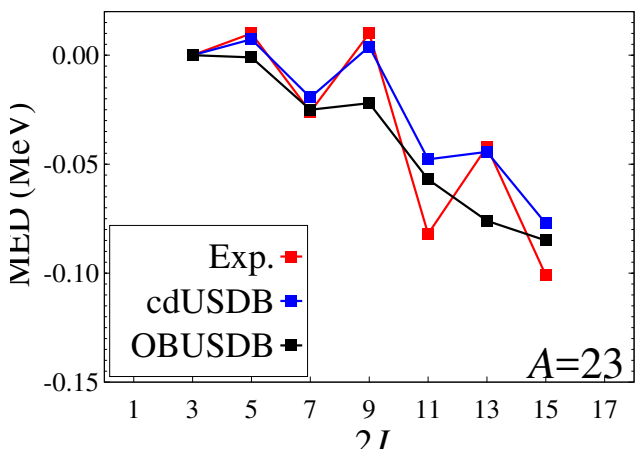

Figure 3. The MED of mass $A=23$. Experimental data are quoted from Refs. [19, 20]. 


\section{Conclusions}

We have used a recently constructed INC $s d$-shell Hamiltonian based on USDB to describe a partial-decay scheme of ${ }^{24} \mathrm{Si}$ and MEDs in $T=1 / 2$ mirror $A=21$ and $A=23$ nuclei. The results are compared with another INC Hamiltonian, OBUSDB, comprised from an earlier parameterization of charge-dependent terms on top of the USDB interaction. We found that while the USDB interaction well describes the $\beta$-decay lifetime and the GT strength distribution, the INC part robustly reproduces the measured isospin-forbidden proton branching ratios. In addition, we find that both Hamiltonians can reconstruct reasonably well the trends of MEDs in a few $s d$-shell $T=1 / 2$ isobaric mirror pairs, while MEDs for some states stay a challenge. It would be interesting and important to pursue this study in order make a link to the necessity of a $J$-dependent matrix element.

\section{References}

[1] Y. H. Lam, N. A. Smirnova, E. Caurier, Phys. Rev. C 87, 054304 (2013).

[2] B. A. Brown and W. A. Richter, Phys. Rev. C 74, 034315 (2006).

[3] R. Roth and T. Neff and H. Feldmeier, Prog. Part. Nucl. Phys. 65, 50 (2010).

[4] Y. H. Lam et al., At. Data Nucl. Data Tables 99, 680 (2013).

[5] B. A. Brown and W. D. M. Rae, Nucl. Data Sheets 120, 115 (2014).

[6] B. Blank and M. J. G. Borge, Prog. Part. Nucl. Phys. 60, 403 (2008).

[7] B. A. Brown, Phys. Rev. Lett. 65, 2753 (1990).

[8] N. A. Smirnova et al., Phys. Rev. C 93, 044305 (2016).

[9] S. Czajkowski et al., Nucl. Phys. A 628, 537 (1998).

[10] V. Banerjee et al., Phys. Rev. C 63, 024307 (2001).

[11] Y. Ichikawa et al., Phys. Rev. C 80, 044302 (2009).

[12] Y. Ichikawa et al., J. Phys.: Conf. Ser. 312, 092031 (2011).

[13] W. E. Ormand and B. A. Brown, Nucl. Phys. A 491, 1 (1989).

[14] W. A. Richter, S. Mkhize, B. A. Brown, Phys. Rev. C 78, 064302 (2008).

[15] Y. H. Lam, L. Xayavong, N. A. Smirnova, B. Blank, in preparation.

[16] M. A. Bentley, S. M. Lenzi, Prog. Part. Nucl. Phys. 59, 497 (2007).

[17] S. M. Lenzi and M. A. Bentley, Test of Isospin Symmetry Along the $N=Z$ Line, in: J. S. Al-Khalili, E. Roeckl (eds) The Euroschool Lectures on Physics with Exotic Beams, Vol. III, Lect. Notes Phys. 764, 57 (2009), Springer Berlin, Heidelberg.

[18] National Nuclear Data Center (NNDC) online, http://www.nndc.bnl.gov/ensdf

[19] D. G. Jenkins et al., Phys. Rev. C 87, 064301 (2013).

[20] A. Boso et al., Acta Phys. Pol. B 48, 313 (2017).

[21] A. P. Zuker et al., Phys. Rev. Lett. 89, 142502 (2002). 